\title{
Network cohesion
}

\author{
Tiago V. V. Cavalcanti ${ }^{1}$. Chryssi Giannitsarou ${ }^{1}$ (D) \\ Charles R. Johnson ${ }^{2}$
}

Received: 17 September 2015 / Accepted: 24 June 2016 / Published online: 11 July 2016 (C) The Author(s) 2016. This article is published with open access at Springerlink.com

\begin{abstract}
We define a measure of network cohesion and show how it arises naturally in a broad class of dynamic models of endogenous perpetual growth with network externalities. Via a standard growth model, we show why network cohesion is crucial for conditional convergence and explain that as cohesion increases, convergence is faster. We prove properties of network cohesion and define a network aggregator that preserves network cohesion.
\end{abstract}

Keywords Network theory $\cdot$ Endogenous growth models · Convergence $\cdot$ Consensus

Mathematics Subject Classification D85 - O40 - D62

\section{Introduction}

The purpose of this paper is to propose an aggregate measure for networks that is useful for analyzing models of endogenous growth with network externalities. As an example, we present a standard endogenous growth model in which the productivity of a particular country (or region) depends on the average stock of capital of its neighbor countries (or regions). Generally, without such externalities (i.e., when the network is empty and cohesion is zero), this model predicts that there will be no crosscountry conditional convergence of income. However, under a certain condition, we can establish conditional convergence of all countries, and that convergence to the long-run balanced growth path is faster when network cohesion is higher.

\footnotetext{
$\bowtie$ Chryssi Giannitsarou cg349@cam.ac.uk

1 University of Cambridge, Faculty of Economics, Cambridge CB3 9DD, UK

2 College of William and Mary, P.O. Box 8795, Williamsburg, VA 23187-8795, USA
} 
The condition for convergence requires that the underlying network of externalities satisfies a certain property, which naturally defines the measure that we introduce. This novel summary statistic for a (possibly directed and weighted) network can be interpreted as a measure of network cohesion. It is defined via a matrix of dispersion which has typical elements that represent the deviation of intensity of the links from node $j$ to node $i$, from the average intensity of the links from node $j$. The elements of this matrix become larger in absolute value as the intensity of links between nodes becomes more uneven, and the network cohesion is then defined as one minus the largest modulus eigenvalue of this matrix. This cohesion measure effectively gives a characterization of how uniform or fragmented a network is. We show that it is independent of how we label the nodes, and that it varies between zero and one. The complete network has the largest possible network cohesion, equal to 1 , while the empty network (or any disconnected network) has the lowest cohesion, equal to 0.

Although we motivate network cohesion using a simple growth model, we argue that this statistic is relevant for characterizing the stability and the speed of convergence of any similar dynamic system with spillover effects from neighbors, in which a balanced growth path, rather than a steady state, is present, and analysis is carried out in terms of relative variables of interest, e.g., physical capital, human capital, beliefs and prices, divided by the average value of the corresponding variable in the whole system. It is in this sense that network cohesion is useful for analysis of a large variety of endogenous growth models with an underlying network structure and more broadly important for a growing literature on networks in economics. ${ }^{1}$

After carefully defining network cohesion and characterizing some of its properties, we also propose a way in which large networks can be aggregated into smaller networks that share the same cohesion. In practice, this aggregation technique can be implemented in a straightforward manner and is helpful for reducing models with many dimensions to smaller, more manageable systems, without losing the dynamic properties of the original system. Last, we provide a detailed comparison between network cohesion to two other network statistics that are related to it, namely algebraic connectivity (see Fiedler 1973 and Mohar 1991) and the recently introduced spectral homophily (see Golub and Jackson 2012a, b). We also explain how network cohesion is related to other aggregate network measures that capture cohesion.

Our contribution is broadly related to a large variety of literature within engineering, biology and political economy, whenever the common factor between these and our setting is a system of variables with dynamics that evolve via an average-based updating process (i.e., a process by which next period's node-specific variable depends on the current average of such a variable in the node's neighborhood). For example, in our growth model setting, the future capital of a country depends on the average capital of the countries that this country is linked to (via trade and technological spillovers). A more detailed discussion of how the present work fits into the literature is presented at the end of Sect. 4.

1 Goyal (2007) and Jackson (2008) provide an overview of recent research and models on networks in economics and techniques for analyzing different economic issues. Network cohesion is used for the analysis of an endogenous growth model with human capital in Cavalcanti and Giannitsarou (2015). 
There is an important difference between the setting we are interested in and other related strands of literature that focus on dynamic processes that converge to a stationary, constant steady state (i.e., convergence of Markov processes and convergence of beliefs). In such settings it is known that convergence and its speed depend on the second largest modulus eigenvalue of a stochastic matrix with typical element that gives the intensity of the links from node $j$ to node $i$. We, on the other hand, are interested in growth models, where perpetual endogenous growth implies convergence to a balanced growth path rather than to a constant steady state. In such growth models, the dynamics are not stationary and their local properties are determined by a matrix which, as we show, is an appropriate transformation of the matrix that determines the local dynamics of the corresponding detrended model.

Our main contributions are then to (a) demonstrate that the dynamics of such systems cannot be analyzed directly by using known network measures such as algebraic connectivity or spectral homophily, (b) show how the dynamics of such systems reduce to a matrix that is closely related to the corresponding stochastic (intensity) matrix of a system with a steady state and (c) show formally that the spectrum of this matrix is the same as the spectrum of the corresponding stochastic matrix after replacing eigenvalue 1 with eigenvalue 0 . These results not only allow us to show properties of network cohesion and give statements regarding convergence and speed of the dynamic systems we study, but also emphasize the fact that network cohesion can be thought of as one minus the second largest modulus eigenvalue of the stochastic matrix that corresponds to a system with a steady state. We discuss these points in more detail in Sects. 4 and 6 , where we also refer to specific strands of literature and various related papers.

\section{A motivating example}

We present a very basic endogenous growth model with technological spillovers. We assume that there are $n$ countries (or regions and states) which are the nodes of a network $N$. Let $g_{i j} \in\{0,1\}$ be a relationship between two countries $i$ and $j$. For this example, it is assumed that $g_{i j}=g_{j i}$ (i.e., that the network is undirected) and that $g_{i j}=1$, if there is a link between regions $i$ and $j$ and $g_{i j}=0$ otherwise. We also assume that $g_{i i}=1$. This notation allows us to represent the links in the network with an adjacency matrix $G .^{2}$ The interpretation of such links between two countries $i$ and $j$ may be trade between them, or simply geographical proximity, e.g., the two countries sharing a border. The importance of spatial externalities for growth, as in the latter interpretation, has previously been emphasized in the literature, e.g., see Ertur and Koch (2007) and references therein. Network externalities and their importance for growth are also addressed in Konno (2010).

A country $i$ at time $t$ has a production function $y_{i t}=A_{i t} k_{i t}^{\alpha}$, with $0<\alpha<1$, where $y_{i t}$ is output, $k_{i t}$ is capital and $A_{i t}$ is the level of technology of country $i$, which is defined as

\footnotetext{
2 In the literature of networks it is more common to assume no self-links (loops), i.e., $g_{i i}=0$. Here, we adopt the alternative assumption of $g_{i i} \neq 1$, which is more attractive for the types of endogenous growth models that we are interested in.
} 


$$
A_{i t}=A\left(\frac{\sum_{j} g_{i j} k_{j t}}{\sum_{j} g_{i j}}\right)^{\beta} .
$$

with $\beta=1-\alpha$. In other words, technology for country $i$ depends on a constant $A>0$, common to all countries, and on the average capital of this country's neighboring countries. $^{3}$

To keep things as simple as possible, we assume a constant and exogenous savings rate $0<s<1$, no population growth (population is normalized to 1 ) and no exogenous technological change, i.e., $A$ is constant. ${ }^{4}$ Capital accumulates according to

$$
k_{i t+1}=s y_{i t}+(1-\delta) k_{i t},
$$

where $0<\delta \leq 1$ is the depreciation rate.

The standard $A K$ production function can be retrieved under the assumption that the network that describes the technological interdependence of countries is empty, i.e., for $G=I_{n}$. In that case $y_{i t}=A_{i t} k_{i t}^{\alpha}=A k_{i t}$. This benchmark version of the model predicts divergence of countries that have different initial capital (even if all model parameters are identical for all countries). The question we then ask is, if we allow for technological externalities, i.e., any network other than the empty one with $G \neq I_{n}$, can the different countries converge if they have different initial capital, and if yes, how fast? As it turns out, as long as the network is connected (in the graph theoretic sense), there is convergence of the countries to a common balanced growth path and the rate of convergence can be parameterized by the measure of network cohesion that this paper introduces.

Let $\bar{k}_{t}$ be the average capital of all countries at time $t$, and let $x_{i t}=k_{i t} / \bar{k}_{t}$ be the relative capital of country $i$ at time $t$. Then, the dynamic system that describes the economy is given by

$$
x_{i t+1}=\frac{\gamma_{i t} x_{i t}}{\frac{1}{n} \sum_{i} \gamma_{i t} x_{i t}},
$$

in which

$$
\gamma_{i t}=s A\left(\frac{\sum_{j} g_{i j} x_{j t}}{\sum_{j} g_{i j}}\right)^{\beta} x_{i t}^{-\beta}+(1-\delta)
$$

is the growth rate of the capital stock of country $i$ at time $t$. Collecting the dynamic equations for all countries, we can determine the evolution of this system by analyzing the following nonlinear system of difference equations:

$$
\mathbf{x}_{t+1}=\mathbf{W}\left(\mathbf{x}_{t}\right) .
$$

where $\mathbf{W}$ is defined via (3) and (4). It is straightforward to verify that $\mathbf{x}^{*}=e_{n} \equiv$ $(1,1, \ldots, 1)^{T}$ is a solution to the system. If this solution is stable, then there is convergence of the initially different countries, since $\mathbf{x}^{*}=e_{n}$ implies that in the

\footnotetext{
3 Here, the term 'neighboring' refers to the countries that country $i$ is linked to in the network.

4 A microfounded version of this model would be an overlapping generations economy. This would also yield a constant savings rate that depends on the discount factor.
} 
balanced growth path, $k_{i t}=k_{t}^{*}$ for all countries irrespective of the initial conditions. Moreover, along this balanced growth path all countries will grow at the same rate $\gamma^{*}=\frac{k_{t+1}^{*}}{k_{t}^{*}}=s A+(1-\delta)$.

To establish whether the solution is stable, we need to evaluate the Jacobian

$$
J(\mathbf{x})=\frac{\mathrm{d} \mathbf{W}(\mathbf{x})}{\mathrm{d} \mathbf{x}}
$$

at $\mathbf{x}^{*}=e_{n}$. For $i, j=1, \ldots, n$, let

$$
f_{i j}=\frac{g_{i j}}{\sum_{m=1}^{n} g_{i m}}-\frac{1}{n} \sum_{i=1}^{n} \frac{g_{i j}}{\sum_{m=1}^{n} g_{i m}} .
$$

Then, we get

$$
J\left(\mathbf{x}^{*}\right)=\frac{1}{s A+1-\delta}\left[\beta s A F+(\alpha s A+1-\delta) C_{n}\right],
$$

in which $F$ is a matrix with typical element $f_{i j}$ and $C_{n}=I_{n}-\frac{1}{n} e_{n} e_{n}^{T}$, with $I_{n}$ the $n \times n$ identity matrix and $e_{n}$ an $n \times 1$ column vector of ones. The stability of the solution is therefore determined by the eigenvalues of $J\left(\mathbf{x}^{*}\right)$. If all eigenvalues of $J\left(\mathbf{x}^{*}\right)$ are inside the unit circle, then the system is stable and the countries converge. Moreover, the smaller the eigenvalues of $J\left(\mathbf{x}^{*}\right)$ are in absolute value, the faster convergence is.

The measure of network cohesion we propose is defined as one minus the largest modulus eigenvalue of $F$ and arises naturally in this simple growth model. As we will show in Sect. 4, nonzero network cohesion ensures convergence of the countries and the higher it is, the faster convergence will be. With this example in mind, we propose a general definition of network cohesion in the next section and then explain why this statement is true.

\section{A measure of network cohesion}

The network $N$, which can generally be weighted and/or directed, consists of a set of nodes labeled by $i=1,2, \ldots, n$. It is assumed that $g_{i j}>0$ if there is a link from node $j$ to node $i$ and $g_{i j}=0$ otherwise. We adopt the convention that $g_{i i}>0$, for all $i=1,2, \ldots, n$. This notation allows us to represent the network by the adjacency matrix $G=\left[g_{i j}\right] .^{5}$

\footnotetext{
5 If the network is undirected, as in the growth model of Sect. 2, then the matrix $G$ is symmetric. If the network is unweighted, then $g_{i j}=1$ if there is a link between $i$ and $j$, and $g_{i i}=1$ for all $i$. Allowing for weights that are generally different from one or for nonsymmetric adjacency matrices is very general and allows us to consider, for example, growth models in which the spillover effects may be stronger between some countries and weaker between others. The magnitude of weights in the diagonal elements may reflect the importance of a country's own capital for its productivity, relative to the remaining countries' capital.
} 
Next, let

$$
D=\operatorname{diag}\left[\sum_{j} g_{1 j}, \sum_{j} g_{2 j}, \ldots, \sum_{j} g_{n j}\right],
$$

and define the row stochastic matrix

$$
R=D^{-1} G
$$

with typical element

$$
r_{i j}=\frac{g_{i j}}{\sum_{j} g_{i j}}
$$

that represents the intensity of the link from node $j$ to node $i .{ }^{6}$

Finally, let

$$
C_{n}=I_{n}-\frac{1}{n} e_{n} e_{n}^{T}
$$

be the centering matrix of order $n .^{7}$ We can now define the matrix

$$
F=C_{n} R \text {. }
$$

We call $G$ the adjacency matrix, $R$ the intensity matrix and $F$ the relative intensity matrix. We now provide the mathematical definition of our measure of network cohesion.

Definition 1 Given a network $N$ with adjacency matrix $G$, let matrices $C_{n}, R$ and $F$ be defined as in (10), (12) and (13). The network cohesion is defined as $\kappa \equiv 1-\rho(F)$, in which $\rho(F)$ is the spectral radius of the matrix $F$.

We next explain informally what this measure captures. We first look at a typical element of the intensity matrix, $r_{i j}$. This represents how 'intense' the link from node $j$ to node $i$ is. For example, for a undirected, unweighted network, the intensity of every link of node $i$ is the inverse of the node's degree (i.e., if node $i$ has three links, each link is one-third intense). If the network is weighted, the intensity for node $i$ is the weight of the link with node $j$, over of the strength of node $i$, and it is larger for nodes $j$ that have high weights in the adjacency matrix. In other words, the intensity matrix represents a normalization of the adjacency matrix. Going back to the typical element $r_{i j}$, suppose now that we want to compare the intensity $r_{i j}$ of the link from $j$ to $i$, with the average intensity from node $j$ to other nodes of the network; we can then calculate $f_{i j}=r_{i j}-\bar{r}_{j}$ (with $\bar{r}_{j}=\left(\sum_{i} r_{i j}\right) / n$ ), and call this the relative intensity of the link from node $j$ to node $i$. If $f_{i j}>0$, this means that node $i$ has a more intense relationship to $j$ than other nodes have with $j$ on average, while if $f_{i j}<0$, node $i$ has a less intense relationship to $j$ than other nodes have with $j$ on average. It is easy to

\footnotetext{
6 The matrix $R$ is also used in Echenique and Fryer (2007), where it is called the matrix of social interactions.

7 The term centering is borrowed from statistics. The matrix $C_{n}$ is a symmetric and idempotent matrix that, when multiplied with a vector, has the same effect as subtracting the mean of the elements of the vector from every vector entry.
} 
see that generally, if the elements $f_{i j}$ are close to zero, i.e., if they do not deviate much from the averages, the nodes and relationships (links) in the network are alike, then the spectral radius $\rho(F)$ is small, and the network is cohesive. If the elements $f_{i j}$ are far from zero, i.e., if the elements $f_{i j}$ are large in absolute value, the more variability there is in the intensity of links in the network, and the spectral radius $\rho(F)$ is large. In the context of the simple growth model presented in Sect. 2, the typical element $f_{i j}$ of the matrix $F$ represents how important the spillover/externality from country $j$ to country $i$ is, relative to the average spillover from country $j$ to the rest of the world. It is in this sense that the matrix $F$ can be interpreted as a matrix of dispersion, and therefore we call the spectral radius $\rho(F)$ of matrix $F$ the coefficient of dispersion of the network.

High variability among the different $f_{i j}$ s can also be interpreted as the network having more uneven, unequal interactions, i.e., less cohesion in the network. Consider, for example, two extreme cases, first, an undirected network, in which everyone is linked to each other (i.e., the complete network), and second the case of an empty undirected network. In the former case, the interaction with $j$ is of importance $1 / n$ for node $i$, and that is true for all nodes $i$, so that the average $\bar{r}_{j}$ is also $1 / n$. Therefore, all the elements of $F$ are zero, and $\kappa=1$, i.e., there is the highest possible network cohesion. In the latter case, there is no interaction (link) between any two nodes and $\kappa=0 .{ }^{8}$

Next, we provide two basic important properties of $\kappa$. The first states that isomorphic networks have the same cohesion and the second states that the cohesion of a network is always between zero and one. Both these two results hinge on the following theorem, which we state and prove first.

Theorem 1 If a matrix $Q \in \mathbb{R}^{n \times n}$ has rows that sum to one, then

$$
\rho\left(C_{n} Q\right)=\max _{\lambda \in Z}|\lambda|
$$

in which $Z=\sigma(Q) \backslash\{1\}$, and $\sigma(Q)$ is the set of eigenvalues of $Q$.

Proof First, note that 0 is an eigenvalue of the centering matrix $C_{n}$ associated with eigenvector $e_{n}$. This is true because

$$
C_{n} e_{n}=\left(I_{n}-\frac{1}{n} e_{n} e_{n}^{T}\right) e_{n}=e_{n}-\frac{1}{n} e_{n} n=0 .
$$

Also, recall that for any matrix with rows that sum to one, 1 is an eigenvalue associated with eigenvector $e_{n}$. Now let

$$
S_{n}=\left(\begin{array}{cc}
1 & 0_{n-1}^{T} \\
e_{n-1} & I_{n-1}
\end{array}\right)
$$

with

$$
S_{n}^{-1}=\left(\begin{array}{cc}
1 & 0_{n-1}^{T} \\
-e_{n-1} & I_{n-1}
\end{array}\right)
$$

\footnotetext{
8 Note that applying the centering matrix $C_{n}$ to $R$ from the left removes the mean from each of the columns of a matrix, therefore collecting the elements $f_{i j}=r_{i j}-\bar{r}_{j}$ into a matrix $F$ gives the same elements as $C_{n} R$.
} 
and observe that the first column of matrix $S_{n}$ is the eigenvector of $C_{n}$ corresponding to eigenvalue 0 and the eigenvector of $Q$ corresponding to eigenvalue 1 . Then

$$
S_{n}^{-1} C_{n} S_{n}=\left(\begin{array}{cc}
0 & -\frac{1}{n} e_{n-1}^{T} \\
0 & I_{n-1}
\end{array}\right)
$$

From Horn and Johnson (1985), ex. 8 in 1.4 (matrix deflation), we have that the remaining eigenvalues of $C_{n}$ are those of the matrix in the lower right block of the above matrix, i.e., 1 with algebraic multiplicity $n-1$. Moreover,

$$
S_{n}^{-1} Q S_{n}=\left(\begin{array}{cc}
1 & * \\
0 & Q_{1}
\end{array}\right)
$$

where from the same result we have that the remaining eigenvalues of $Q$ are those of $Q_{1}$, i.e., $\sigma\left(Q_{1}\right)=\sigma(Q) \backslash\{1\}$. Next, we will show that $\sigma\left(C_{n} Q\right)=(\sigma(Q) \backslash\{1\}) \cup$ $\{0\}$, i.e., that the spectrum of $C_{n} Q$ is generated by replacing eigenvalue 1 with 0 in the spectrum of $Q$. First, it is straightforward to show that 0 is an eigenvalue of $C_{n} Q$, with eigenvector $e_{n}$ : Since $Q e_{n}=e_{n}$, we have that

$$
C_{n} Q e_{n}=\left(I_{n}-\frac{1}{n} e_{n} e_{n}^{T}\right) Q e_{n}=\left(I_{n}-\frac{1}{n} e_{n} e_{n}^{T}\right) e_{n}=e_{n}-\frac{1}{n} e_{n} n=0 .
$$

Then, using the same result as above, we can use the same $S_{n}$ to show that

$$
S_{n}^{-1} C_{n} Q S_{n}=\left(S_{n}^{-1} C_{n} S_{n}\right)\left(S_{n}^{-1} Q S_{n}\right)=\left(\begin{array}{cc}
0 & -\frac{1}{n} e_{n-1}^{T} Q_{1} \\
0 & Q_{1}
\end{array}\right) .
$$

Therefore the remaining eigenvalues of $C_{n} Q$ are the eigenvalues of $Q_{1}$, i.e., all the eigenvalues of $Q$ apart from 1 . In other words,

$$
\sigma\left(C_{n} Q\right)=(\sigma(Q) \backslash\{1\}) \cup\{0\}
$$

This implies that

$$
\rho\left(C_{n} Q\right)=\max _{\lambda \in Z}|\lambda|
$$

This theorem says that if we center a matrix $Q$ with rows that sum to one around its column means, then the spectral radius of the centered matrix is the second largest modulus eigenvalue of $Q$. In fact, the theorem shows a lot more than that, i.e., that the whole spectrum of $C_{n} Q$ coincides with the spectrum of $Q$ after replacing eigenvalue 1 with eigenvalue 0 . Note that this theorem holds generally for matrices with rows that sum to one, not just stochastic matrices. An immediate consequence of this theorem is that the cohesion of a network is equal to one minus the second largest modulus eigenvalue of its intensity matrix. This observation is very useful for proving properties of network cohesion. With this result in place, we can now show two properties of network cohesion $\kappa$. 
Proposition 1 Isomorphic networks have the same cohesion.

Proof Let $N_{1}$ and $N_{1}$ be networks with adjacency matrices $G_{1}$ and $G_{2}$, respectively. Since the two networks are isomorphic, there exists a permutation matrix $P$ such that $G_{2}=P G_{1} P^{-1}$. Also, $P^{-1}=P^{T}$ since $P$ is a permutation matrix. Let $R_{1}=D_{1} G_{1}$ and $R_{2}=D_{2} G_{2}$ be their corresponding intensity matrices. To show the result it suffices to show that $R_{1}$ and $R_{2}$ have the same eigenvalues. Note that

$$
R_{2}=D_{2} G_{2}=D_{2} P G_{1} P^{T}=P D_{1} G_{1} P^{T}=P R_{1} P^{T}
$$

where the third equality follows the fact that if $G_{2}=P G_{1} P^{-1}$, then it must be that $D_{2}=P D_{1} P^{-1}$ from (9). Therefore $R_{1}$ and $R_{2}$ are similar and thus have the same eigenvalues.

Proposition 2 The cohesion of a network $N$ satisfies the inequality $0 \leq \kappa \leq 1$.

Proof From the definition of the network cohesion and Theorem 1, it suffices to show that the second largest modulus eigenvalue of $R$ is between 0 and 1. From Theorem 8.1.22 of Horn and Johnson (1985), we have that

$$
\min _{i} \sum_{j} r_{i j} \leq \rho(R) \leq \max _{i} \sum_{j} r_{i j} \Longleftrightarrow 1 \leq \rho(R) \leq 1 .
$$

Therefore, the spectral radius of $\rho(R)$ is exactly equal to 1 . It follows that, $|\lambda| \leq$ $\rho(R)=1$ for any other eigenvalue $\lambda$ of $R$.

Corollary 1 Special networks

(i) For the empty network the cohesion is $\kappa=0$.

(ii) For the complete network the cohesion is $\kappa=1$.

(iii) For the star network the cohesion is $\kappa=1 / 2$.

Proof All three statements follow immediately by applying Theorem 1 and Proposition 1 to the particular networks.

Corollary 2 Disconnected networks have zero cohesion.

Proof Without loss of generality, we consider a disconnected network that consists of two components. Then from Proposition 1, there exists an isomorphic network with the same cohesion and has intensity matrix that can be written in the block form

$$
R=\left(\begin{array}{cc}
R_{1} & 0 \\
0 & R_{2}
\end{array}\right)
$$

where $R_{1}$ and $R_{2}$ are the corresponding intensity matrices of the two disconnected components. The eigenvalues of $R$ are then all the eigenvalues of $R_{1}$ and $R_{2}$. To see this let for example $\lambda_{1}$ be an eigenvalue of $R_{1}$, with eigenvector $x_{1}$. Then

$$
\left(\begin{array}{cc}
R_{1} & 0 \\
0 & R_{2}
\end{array}\right)\left(\begin{array}{c}
x_{1} \\
0
\end{array}\right)=\left(\begin{array}{c}
R_{1} x_{1} \\
0
\end{array}\right)=\left(\begin{array}{c}
\lambda_{1} x_{1} \\
\lambda_{1} 0
\end{array}\right)=\lambda_{1}\left(\begin{array}{c}
x_{1} \\
0
\end{array}\right),
$$


i.e., $\lambda_{1}$ is an eigenvalue of $R$ with eigenvector $x=\left(x_{1}^{T} 0^{T}\right)^{T}$. Since each of the intensity matrices $R_{1}$ and $R_{2}$ has 1 as an eigenvalue, $R$ has 1 as an eigenvalue with multiplicity at least 2 . Therefore, from Theorem 1 , after replacing one of these with the zero eigenvalue, the set of eigenvalues of $F$ still contains 1 , and therefore, $\kappa$ $=0$.

Proposition 2 states that network cohesion varies between 0 and 1 and Corollary 1 gives a natural interpretation to these two bounds, by mapping them to two extreme networks: the smallest possible cohesion is achieved for the empty network (no links), and the highest cohesion is achieved for the complete network (all links). The star network has cohesion equal to $1 / 2$ and provides a separation point between networks with low cohesion $(<1 / 2)$ and high cohesion $(>1 / 2)$.

\section{AK growth model continued and some other examples}

We now return to the simple growth model that we presented in Sect. 2. In order to determine under what conditions the system (5) converges to $\mathbf{x}^{*}=e_{n}$, and at what speed, we first show an auxiliary result that provides the exact spectrum of the Jacobian at $\mathbf{x}^{*}$.

Lemma 1 For a network $N$, let $F$ be the relative intensity matrix and $C_{n}$ as defined in Sect. 3. Then, for any scalars $b, d \in \mathbb{R}$, the spectrum of $b F+d C_{n}$ is

$$
\sigma\left(b F+d C_{n}\right)=\left\{b \lambda_{1}+d, \ldots, b \lambda_{n-1}+d, 0\right\}
$$

with $\lambda_{i} \in \sigma(F) \backslash\{0\}$.

Proof First note that $e_{n} e_{n}^{T} F=0$, and $F e_{n} e_{n}^{T}=0$, and therefore

$$
F C_{n}=F-F\left(\frac{1}{n} e_{n} e_{n}^{T}\right)=F-\left(\frac{1}{n} e_{n} e_{n}^{T}\right) F=C_{n} F,
$$

i.e., the matrices $F$ and $C_{n}$ commute. From Theorem 2.4.9 in Horn and Johnson (1985), the spectrum of the sum of commuting matrices consists of combinations of sums of the eigenvalues of these matrices, i.e., $\sigma\left(b F+d C_{n}\right) \subseteq b \sigma(F)+d \sigma\left(C_{n}\right)$. The eigenvalues of $C_{n}$ are 0 with multiplicity 1 and 1 with multiplicity $n-1$, i.e., $\sigma\left(C_{n}\right)=$ $\{0,1, \ldots, 1\}$, and the eigenvalues of $F$ are 0 with multiplicity 1 and $\lambda_{1}, \ldots, \lambda_{n-1}$, i.e., $\sigma(F)=\left\{0, \lambda_{1}, \ldots, \lambda_{n-1}\right\}$, as shown in Theorem 1. For both $C_{n}$ and $F$ the eigenvector corresponding to eigenvalue 0 is $e$. This implies that 0 is also an eigenvalue of $b F+d C_{n}$ with eigenvector $e$. We can then apply the same deflation argument as in the proof of Theorem 1, with $S_{n}$ as defined in expression (16), to obtain

$$
S_{n}^{-1}\left(b F+d C_{n}\right) S_{n}=b\left(\begin{array}{cc}
0 & * \\
0 & F_{1}
\end{array}\right)+d\left(\begin{array}{cc}
0 & * \\
0 & I_{n-1}
\end{array}\right)=\left(\begin{array}{cc}
0 & * \\
0 & b F_{1}+d I_{n-1}
\end{array}\right) \text {. }
$$

Then, the remaining eigenvalues of $b F+d C_{n}$ are the eigenvalues of $b F_{1}+d I_{n-1}$, i.e., $b \lambda_{1}+d, \ldots, b \lambda_{n-1}+d$. Therefore

$$
\sigma\left(b F+d C_{n}\right)=\left\{b \lambda_{1}+d, \ldots, b \lambda_{n-1}+d, 0\right\} .
$$


We can now establish the following result for the $A K$ growth model with technology spillovers:

Proposition 3 Let the dynamics of the $n$ countries in network $N$ be described by (5). Then the solution $\mathbf{x}^{*}=e_{n}$ is stable and the countries converge, if the network cohesion is strictly positive, i.e., if $\kappa>0$.

Proof To confirm that the solution is stable, we need to examine whether the eigenvalues of the Jacobian at $\mathbf{x}^{*}$ are inside the unit circle. Recall that

$$
J\left(\mathbf{x}^{*}\right)=\frac{1}{s A+1-\delta}\left[\beta s A F+(\alpha s A+1-\delta) C_{n}\right] .
$$

Using Lemma 1, we can establish that the eigenvalues of $J\left(\mathbf{x}^{*}\right)$ are

$$
\left\{b \lambda_{1}+d, \ldots, b \lambda_{n-1}+d, 0\right\}
$$

where

$$
b=\frac{\beta s A}{s A+1-\delta}>0, d=\frac{\alpha s A+1-\delta}{s A+1-\delta}>0 .
$$

For the solution $\mathbf{x}^{*}=e$ to be stable, we need all the eigenvalues of $J\left(\mathbf{x}^{*}\right)$ to be strictly inside the unit circle, i.e., we need $\left|b \lambda_{i}+d\right|<1$. First we note that the eigenvalues of $F$ are real, because they coincide with the eigenvalues of the row stochastic matrix $R$ apart from eigenvalue $\lambda_{0}=1$, as shown in Theorem 1 , and $R$ is similar to $D^{-1 / 2} G D^{-1 / 2}$, which is symmetric for undirected networks. We also know that $0 \leq\left|\lambda_{i}\right| \leq 1$ since $R$ is row stochastic. From the triangle inequality, and since $b, d \geq 0$, a sufficient condition for stability is then $b\left|\lambda_{i}\right|+d<1$. Recalling that $\beta=1-\alpha$, the condition is equivalent to requiring that $\left|\lambda_{i}\right|<1$ for all $i$, or that $\kappa=1-\rho(F)>0$.

The intermediate steps of the above proof also provide insights about the rate of convergence in this growth model. The spectrum of the Jacobian is

$$
\sigma\left(J\left(\mathbf{x}^{*}\right)\right)=\left\{\frac{\beta s A}{s A+1-\delta} \lambda_{i}+\frac{\alpha s A+1-\delta}{s A+1-\delta}, i=1, \ldots, n-1\right\} \cup\{0\} .
$$

Therefore the rate of convergence $\rho\left(J\left(\mathbf{x}^{*}\right)\right)$ for (5) cannot be higher than $1-\frac{(1-\alpha) s A}{s A+1-\delta} \kappa$, an upper bound that decreases as cohesion increases and the following is true:

Corollary 3 Higher network cohesion implies faster convergence to the balanced growth path of the balanced growth model of Sect. 2.

Next, we discuss some other examples in which network cohesion is relevant. First, the measure of network cohesion also arises in Cavalcanti and Giannitsarou (2015) in a model of endogenous growth with overlapping generations and human capital accumulation that depends on investment in education and local network externalities. In that framework, different households represent the nodes of the network. The 
paper then examines under what conditions populations that are initially heterogeneous can converge to a balanced growth path of long-run equality. It is shown that network cohesion is crucial for ensuring stability of the dynamic system and convergence to a balanced growth path of long-run equality, even when the network is connected: if network cohesion is low, the local externality effect may not be strong enough to guarantee convergence to equality, and inequality persists perpetually.

More generally, cohesion as a network statistic is particularly useful in nonstationary dynamic models in which balanced growth, rather than a steady state, may be present. As is typical in such models, the variables and dynamics are transformed by dividing through by averages, and the analysis is carried out in terms or relative variables (i.e., deviations of variables from an average). In such models, the local dynamics and their properties can be approximated at first order by the Jacobian, which is typically a linear function of a dispersion matrix $F$, i.e., a matrix of deviations of network intensities from corresponding averages. The spectral radius of $F$ (and thus network cohesion) is then crucial for determining local stability of a balanced growth path. Then, one can think of a wide spectrum of models in which externalities and neighborhood effects may matter for long-run growth and inequality, and for which network cohesion would be crucial for stability and speed of convergence to a balanced growth path (e.g., Ray 2006; Garcia-Peñalosa and Turnovsky 2008; Mierau and Turnovsky 2014).

These examples are also useful for demonstrating the link between network cohesion and various other strands of literature in engineering, operations research and graph theory. In particular, it is known that the second largest eigenvalue of an appropriately defined stochastic matrix represents the rate of convergence of Markov chains to stationarity and this property has been used to analyze convergence rates of genetic algorithms, various models of statistical physics, or problems of global clock synchronization. Examples of such applications can be found in Mitra and Weiss (1980), Landau and Odlyzko (1981), Diaconis and Stroock (1991), Diaconis and Saloff-Coste (1993), Römer (2001) and Li and Rus (2006). The measure of network cohesion we present here is defined as one minus the coefficient of dispersion, where, as we have shown, the coefficient of dispersion coincides with the second largest modulus eigenvalue of a stochastic matrix. The fact that higher cohesion (or lower coefficient of dispersion) implies faster convergence in dynamic growth models is in direct correspondence with the aforementioned results.

There is also a more direct link to the literature on learning, imitation and social influence in the context of social and information networks (see Goyal (2007) and Jackson 2008, and references therein). In a simple model of consensus formation as in DeGroot (1974), the society starts from initial opinions on a matter. Each agent (in a network) updates his opinion using a weighted average of his current opinion and the average opinion of his neighbors. We can then analyze the dynamic behavior of the vector of all opinions as deviations from the average opinion in the society, and give conditions under which consensus is reached in the limit. Once again, strictly positive network cohesion is required for convergence and the higher cohesion is, the 

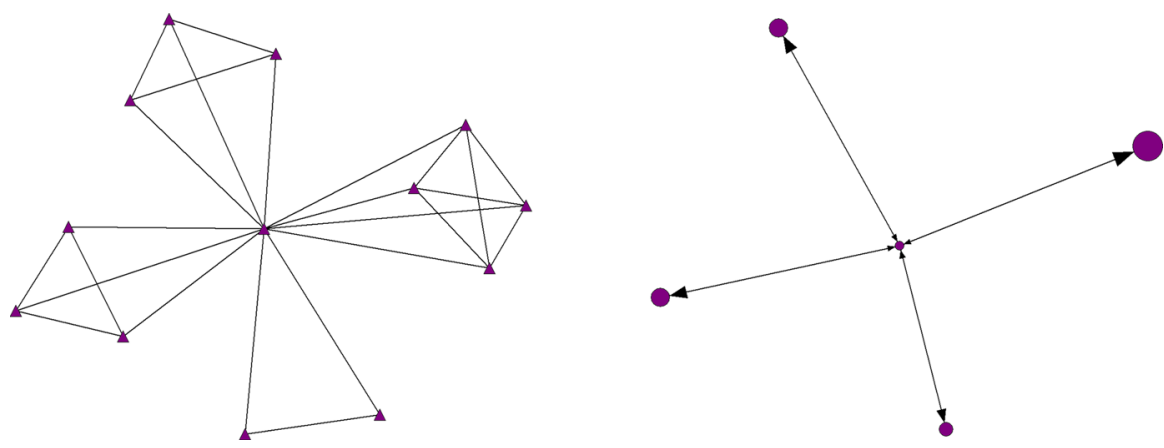

Fig. 1 Network aggregation

faster convergence is achieved. ${ }^{9}$ More generally, our measure of network cohesion can be useful for dynamic settings of opinion formation and disagreement, such as that analyzed in Melguizo (2016).

\section{Aggregation}

In this section, we propose a way of aggregating a large network to a resulting smaller network with the same network cohesion. The importance of such an aggregation result is practical. Its purpose is to reduce a large network structure into a smaller, more manageable setting, while preserving the dynamic properties of their corresponding models. Consider, for example, the context of the AK growth model we have been working with so far, and assume that the world consists of five countries $q=1, \ldots, 5$, each of which have three, two, four, two and one region(s), respectively. We assume that all regions within a country are linked, as shown in the left-hand side of Fig. 1. The adjacency matrix of this large network (the nodes of which are regions) is

$$
G=\left[\begin{array}{ccccc}
e_{3} e_{3}^{T} & 0 & 0 & 0 & e_{3} \\
0 & e_{2} e_{2}^{T} & 0 & 0 & e_{2} \\
0 & 0 & e_{4} e_{4}^{T} & 0 & e_{4} \\
0 & 0 & 0 & e_{3} e_{3}^{T} & e_{3} \\
e_{3}^{T} & e_{2}^{T} & e_{4}^{T} & e_{3}^{T} & 1
\end{array}\right]
$$

and its cohesion is 0.2182 .

We then ask, how should we 'aggregate' the network so that the resulting smaller network has the same cohesion as the original one? The answer is intuitive. We construct a new network that has the same number of nodes as the number of groups we identify (here five countries), and each new node corresponds to one of the countries. The new network is weighted, and the link from node $s$ to node $q$ is weighted as $n_{q} n_{s}$ (i.e., the number of regions in country $q$, times the number of regions in country $s$ ) if

9 Interesting variations of this model can be found in DeMarzo et al. (2003) and more recently JiménezMartínez (2015). 
the two groups were originally connected, and 0 otherwise. The resulting aggregated network is pictured in right-hand side of Fig. 1. Its adjacency matrix is

$$
H=\left[\begin{array}{ccccc}
9 & 0 & 0 & 0 & 3 \\
0 & 4 & 0 & 0 & 2 \\
0 & 0 & 16 & 0 & 4 \\
0 & 0 & 0 & 9 & 3 \\
3 & 2 & 4 & 3 & 1
\end{array}\right]
$$

and its cohesion is 0.2182 .

Then, we can study the growth dynamics of the big, disaggregated system of 13 difference equations with

$$
k_{i t+1}=s A\left(\frac{\sum_{j=1}^{13} g_{i j} k_{j t}}{\sum_{j=1}^{13} g_{i j}}\right)^{\beta} k_{i t}^{1-\beta}+(1-\delta) k_{i t}, \quad i=1, \ldots, 13,
$$

or the smaller, aggregated system of 5 difference equations

$$
k_{q t+1}=s A\left(\frac{\sum_{s=1}^{5} h_{q s} k_{s t}}{\sum_{s=1}^{5} h_{q s}}\right)^{\beta} k_{q t}^{1-\beta}+(1-\delta) k_{q t}, \quad q=1, \ldots, 5,
$$

where the matrices $G$ and $H$ are defined as in (34) and (35). The local dynamics of the large system (regions) are described by

$$
\mathbf{x}_{t+1}-\mathbf{x}^{*}=J\left(\mathbf{x}^{*}\right)\left(\mathbf{x}_{t+1}-\mathbf{x}^{*}\right),
$$

with

$$
J\left(\mathbf{x}^{*}\right)=\frac{1}{s A+1-\delta}\left[\beta s A C_{13} R+((1-\beta) s A+1-\delta) C_{13}\right],
$$

and the local dynamics of the small system (countries) are described by

$$
\chi_{t+1}-\chi^{*}=\tilde{J}\left(\chi^{*}\right)\left(\chi_{t+1}-\chi^{*}\right)
$$

where $\chi$ is the vector of relative capital of all countries and

$$
\tilde{J}\left(\chi^{*}\right)=\frac{1}{s A+1-\delta}\left[\beta s A C_{5} P+((1-\beta) s A+1-\delta) C_{5}\right]
$$

is the Jacobian of the small system. In what follows, we will show general results that the ensure that the two Jacobians have the same nonzero eigenvalues. Since the two networks have the same cohesion, the proof of Proposition 4.2 is the same for either network and the rate of converge for both systems is the same.

We now formalize the notion of aggregation and provide a theorem and corollaries that ensure that all the dynamic properties of these two systems are identical. In particular the theorem ensures that the two Jacobians have the same nonzero eigenvalues 
and the corollaries ensure that all these eigenvalues are real and the two networks have the same cohesion. This means that the proof of Proposition 3 is the same for either network and that the rates of converge of the two systems coincide.

For a given undirected network $N=\left\{v_{i}, i=1,2, \ldots, n\right\}$, using a suitable permutation, we first find a corresponding isomorphic network that reorganizes the nodes in such a way that the resulting adjacency matrix $G$ can be written in block form

$$
G=\left[\begin{array}{cccc}
G_{11} & G_{12} & \cdots & G_{1 k} \\
G_{21} & G_{22} & & G_{2 k} \\
\vdots & & \ddots & \vdots \\
G_{k 1} & G_{k 2} & \cdots & G_{k k}
\end{array}\right],
$$

with

$$
\begin{aligned}
G_{q q} & =\pi_{q q} e_{n_{q}} e_{n_{q}}^{T}, \\
G_{q s} & =\pi_{q s} e_{n_{q}} e_{n_{s}}^{T}, \text { whenever } q \neq s,
\end{aligned}
$$

in which $\pi_{q q}>0$ and $\pi_{q s} \geq 0, q \neq s$ are scalars, and the dimensions of $G_{q s}$ are $n_{q} \times n_{s}$. The assumptions on the weights in the network ensure that the diagonal blocks always have strictly positive entries. The off-diagonal blocks can be zero (when the corresponding groups of nodes are not linked) or have strictly positive entries (when the corresponding groups of nodes are linked). Proposition 1 ensures that the cohesion of the original network $N$ is the same as the cohesion of the one with adjacency matrix $G$. Next, we construct the corresponding aggregate network with $k$ nodes, and corresponding adjacency matrix $H$ which has typical element

$$
h_{q s}=n_{q} n_{s} \pi_{q s} .
$$

With this in place, we can state the following results:

Aggregation. Let $N=\left\{v_{i}, i=1,2, \ldots, n\right\}$ be a network that is isomorphic to a network with adjacency matrix $G$ as defined by (42)-(44) and intensity matrix $R$, and let its corresponding aggregate network $M=\left\{u_{q}, q=1,2, \ldots, k\right\}$ have adjacency matrix $H$ given by (45) and intensity matrix $P$. Then the spectrum of $R$ is equal to the spectrum of $P$ plus the eigenvalue 0 with multiplicity $n-k$.

Proof We start from the intensity matrix of the large network that is isomorphic to $N$, denoted by $R$. Its typical element is

$$
r_{i j}=\frac{g_{i j}}{\sum_{j=1}^{n} g_{i j}}=\frac{g_{i j}}{\sum_{j=1}^{n_{1}} g_{i j}+\sum_{j=n_{1}+1}^{n_{1}+n_{2}} g_{i j}+\cdots+\sum_{j=n_{k-1}+1}^{n_{1}+\cdots+n_{k}} g_{i j}} .
$$

But

$$
\sum_{j=n_{s-1}+1}^{n_{1}+\cdots+n_{s}} g_{i j}=n_{s} \pi_{q s}=\frac{n_{q} n_{s} \pi_{q s}}{n_{q}}=\frac{1}{n_{q}} h_{q s}
$$


Therefore, if row $i$ is in block $G_{q s}$, then

$$
r_{i j}=n_{q} \frac{g_{i j}}{h_{q 1}+h_{q 2}+\cdots+h_{q k}} .
$$

Also,

$$
n_{q} g_{i j}=n_{q} \pi_{q s}=\frac{1}{n_{s}} h_{q s} .
$$

Combining these, we have that if row $i$ is in block $G_{q s}$, then

$$
r_{i j}=\frac{1}{n_{s}}\left(\frac{h_{q s}}{\sum_{s=1}^{k} h_{q s}}\right)=\frac{1}{n_{s}} p_{q s},
$$

in which $p_{q s}$ is the typical element of the intensity matrix $P$ for the aggregated network. We can then write $R$ in block matrix form as

$$
R=\left[\begin{array}{ccc}
R_{11} & \cdots & R_{1 k} \\
\vdots & \ddots & \vdots \\
R_{k 1} & \cdots & R_{k k}
\end{array}\right]
$$

where

$$
R_{q s}=p_{q s}\left(\frac{1}{n_{s}} e_{n_{q}} e_{n_{s}}^{T}\right) .
$$

First we show that if $\lambda$ is an eigenvalue of $P$, then it will also be an eigenvalue for $R$. To see this, let $x=\left[x_{1}, \ldots, x_{k}\right]^{T}$ be an eigenvector of $P$, corresponding to eigenvalue $\lambda$, and let $\tilde{x}=\left[\begin{array}{lll}x_{1} e_{n_{1}}^{\prime} & \cdots & x_{k} e_{n_{k}}^{\prime}\end{array}\right]^{\prime}$. Then,

$$
R \tilde{x}=\left[\begin{array}{c}
\left(p_{11} x_{1}+p_{12} x_{2}+\cdots p_{1 k} x_{k}\right) e_{n_{1}} \\
\vdots \\
\left(p_{k 1} x_{1}+p_{k 2} x_{2}+\cdots+p_{k k} x_{k}\right) e_{n_{k}}
\end{array}\right]=\left[\begin{array}{c}
\lambda x_{1} e_{n_{1}} \\
\vdots \\
\lambda x_{k} e_{n_{k}}
\end{array}\right]=\lambda \tilde{x}
$$

The penultimate equality follows from the fact that $\lambda$ is an eigenvalue of $P$ corresponding to eigenvector $x$. Therefore, $k$ of the $n$ eigenvalues of $R$ are the $k$ eigenvalues of $P$. Next, we show that the remaining eigenvalues are all zero. First, it is trivial to show that the linear system $R y=0$ has infinitely many solutions, with $n-k$ free variables. Therefore, 0 is an eigenvalue, with geometric multiplicity equal to $\operatorname{dim} N u l R$, where $N u l R$ is the null space of $R$. Since the geometric multiplicity is smaller than or equal to algebraic multiplicity of 0 , and there cannot be more than $n$ eigenvalues in total, it must be that the algebraic multiplicity of 0 is $n-k$.

Corollary 4 Let $N=\left\{v_{i}, i=1,2, \ldots, n\right\}$ be a network that is isomorphic to a network with adjacency matrix $G$ as defined by (42)-(44) and let its corresponding aggregate network $M=\left\{u_{q}, q=1,2, \ldots, k\right\}$ have adjacency matrix $H$ given by (45). Then $\kappa_{N}=\kappa_{M}$. 
Proof Follows immediately from Theorems 1 and 5.

Corollary 5 Let $M=\left\{u_{q}, q=1,2, \ldots, k\right\}$ be a network that is derived from aggregating a larger network as described by the adjacency matrices (42)-(44) and (45). Then all the eigenvalues of its intensity matrix and relative intensity matrices are real.

Proof Let $P$ be the intensity matrix of $M$. From Theorem 5, $\sigma(P)=\sigma(R) \backslash X\{0, \ldots$, 0 \}, where $R$ is the intensity matrix of the original undirected and unweighted network, with adjacency matrix $G$ and $D$ defined as in (9). Then the matrix $R=D^{-1} G$ is similar to $D^{-1 / 2} G D^{-1 / 2}$. Since $G$ is symmetric, the eigenvalues of $R$ must be real and the result follows. The eigenvalues of the relative intensity matrix $F$ are also real from the fact that its spectrum is equal to the spectrum of $R$ by replacing eigenvalue 1 with eigenvalue 0 .

We note that the network aggregation proposed here is such that the generated intensity (stochastic) matrix $P$ of the aggregated network is an unbiased regular aggregation of the intensity matrix $R$, in the sense defined in Howe and Johnson (1989). The term regular refers to satisfying two axioms, namely that the aggregator is a linear function and that it assigns each state in the original process to exactly one superstate of the aggregated process. The term unbiased refers to the fact that the aggregation depends only on the partition of the original states and preserves the Markovian property. ${ }^{10}$

\section{Comparison of cohesion with other network summary statistics}

In this section we discuss how network cohesion relates to other summary statistics of networks and graphs and explain why they are similar or how they differ. The first statistic we juxtapose with network cohesion is spectral homophily defined in Golub and Jackson (2012a, b). This is defined in the context of a multitype random network, which consists of $n$ nodes, each of which is of one of $k$ different types. Types $q$ and $s$ are linked with some probability $\pi_{q s}$ and not linked with probability $1-\pi_{q s}$. Collecting these probabilities in a matrix $\Pi$, Golub and Jackson (2012a) then define a matrix $P(\Pi, n)$ with typical element

$$
p_{q s}(\Pi, n)=\frac{h_{q s}(\Pi, n)}{\sum_{s=1}^{k} h_{q s}(\Pi, n)},
$$

with

$$
h_{q s}(\Pi, n)=n_{q} n_{s} \pi_{q s} .
$$

10 These can be verified using

$$
S=\left[\begin{array}{cccc}
e_{n_{1}}^{T} & 0 & & 0 \\
0 & e_{n_{2}}^{T} & & 0 \\
& & \ddots & \\
0 & 0 & & e_{n_{k}}^{T}
\end{array}\right] \text { and } T=\left[\begin{array}{cccc}
\frac{1}{n_{1}} e_{n_{1}} & 0 & & 0 \\
0 & \frac{1}{n_{2}} e_{n_{2}} & & 0 \\
& & \ddots & \\
0 & 0 & & \frac{1}{n_{k}} e_{n_{k}}
\end{array}\right]
$$

and applying Theorems 1 and 2 in Howe and Johnson (1989). 
The matrix $H(\Pi, n)$ with typical element $h_{q s}(\Pi, n)$ is the expected number of links between different types of the random network and $p_{q s}(\Pi, n)$ is then the expected fraction of links that nodes of type $q$ will have with nodes of type $s$. The spectral homophily is then defined as the second largest eigenvalue of $P(\Pi, n)$. Any realized network exhibits more (spectral) homophily if the probabilities in the diagonal of this matrix are higher than the off-diagonal probabilities, i.e., when nodes tend to link more often with the own kind (same type nodes). The close relationship between network cohesion and network homophily becomes clear once we reinterpret the matrix defined by (45) as a matrix of the expected number of links between different types as in (53), by imposing $0 \leq \pi_{q s}<1$. Then, the spectral homophily of the random network (i.e., the second largest eigenvalue of $P(\Pi, n))$ is equal to the second largest eigenvalue of the intensity matrix of the aggregate network, which by Theorem 1 and the aggregation results, is equal to one minus the cohesion of a large network defined by (42)-(44). We note that although the two measures are related, they arise from very different settings: network cohesion on the one hand is not defined for random networks and is in this sense less general; on the other hand, it can be defined for directed and weighted networks, and the aggregation results go through for any $\pi_{q s}>0$, so is in this sense more general than spectral homophily. Also, as emphasized earlier, the way we define network cohesion is suitable for models of endogenous perpetual growth with a balanced growth path; spectral homophily cannot be used directly for such models, without the use of suitable transformations and the mathematical results that we prove here.

The conceptual origins of both network cohesion and spectral homophily can be traced to the second measure we discuss here, namely a normalization of algebraic connectivity introduced by Fiedler (1973) and developed further by Mohar (1991). ${ }^{11}$ This is defined via the graph Laplacian, which is an $n \times n$ matrix $L$, with typical element

$$
l_{i j}=\left\{\begin{array}{cl}
k_{i} & \text { if } i=j \\
-1 & \text { if } i \neq j \text { and } g_{i j} \neq 0 \\
0 & \text { otherwise }
\end{array}\right.
$$

in which $k_{i}$ is the degree of node $i$. The algebraic connectivity is then the second smallest eigenvalue of the Laplacian, and it is nonzero if and only if the network is connected. ${ }^{12}$ It measures how well-connected a network is. In the engineering and biology literature, it is considered a measure of the ability of a network to synchronize: as it becomes larger, the network is better connected and more 'eager' to synchronize. ${ }^{13}$ Given this interpretation, the relation of network cohesion to algebraic connectivity is clearer. To understand this relation, first note that for an undirected and unweighted network, the Laplacian is given by

$$
L=D-G,
$$

\footnotetext{
11 Also see Newman (2010) for further details.

12 The Laplacian is a symmetric matrix, and therefore, all its eigenvalues are real.

13 See, for example, Holroyd (2006).
} 
in which $D$ is defined as in expression (9) and $G$ is the adjacency matrix. Consider a normalization of the Laplacian matrix

$$
\mathcal{L}=D^{-1 / 2} L D^{-1 / 2}=I_{n}-D^{-1 / 2} G D^{-1 / 2},
$$

and recall that $D^{-1 / 2} G D^{-1 / 2}$ is similar to $R$. The eigenvalues of the normalized Laplacian (i.e., a normalized algebraic connectivity) are then equal to 1 minus the eigenvalues of $R$, which in turn will be equal to network cohesion, whenever the second largest modulus eigenvalue of $R$ is nonnegative.

Despite the similarities however, the two statistics are not necessarily identical. First, this relationship between them can be established for undirected networks only, while cohesion can be defined in general for directed networks too. Also, the spectral radius of $F$ is not always the same as the second largest eigenvalue of $R$, if, for example, $R$ has a negative eigenvalue which is larger in absolute value than its second largest eigenvalue, or if the eigenvalues of $F$ are complex. Last, the interpretation of the elements of the matrix $F$ and thus its spectral radius is more natural and straightforward in the context of models with perpetual growth than that of the Laplacian and its normalization.

In the broad literature of graph theory and networks, there is a large variety of measures that capture aspects of cohesion. In the context of social networks, Borgatti et al. (2013) discuss how the term 'cohesion' can be understood and propose a variety of measures that can be of interest when thinking of network cohesion. These include density, average degree, average clustering, various versions of connectivity, number of components, fragmentation and diameter.

We note that while the number of components and fragmentation measures capture cohesion in an intuitive way, they do not provide much information on the internal structure of components. If a network, for example, is even weakly connected, then fragmentation is always zero, and becomes strictly positive only when the network is disconnected. On the other hand, whenever the network is disconnected, our measure of network cohesion is always zero, and becomes strictly positive for (weakly) connected networks. In this sense, our measure is complementary to fragmentation, since $\kappa$ refines our understanding of what happens within components, but assigns a zero to any disconnected network.

Other measures that are can be interpreted broadly as measures of cohesion are assortative mixing by degree, the coefficient of variation, as defined in Acemoglu et al. (2012) and centralization. Figure 2 presents the relationship with some of these to our measure of network cohesion, based on randomly generating 10,000 networks of 13 nodes. Each panel contains a scatter plot of network cohesion (horizontal axis) and the corresponding measure (vertical axis). Definitions for these measures can be found in standard textbooks (e.g., Newman 2010), or the aforementioned references. As expected, as $\kappa$ gets large, combinatorial connectivity, average degree and average clustering increases, while the coefficient of variation broadly decreases. There is no clear relationship between the assortative mixing by degree with network cohesion. The same is true for degree centralization. Of all the measures presented here, the closest one to network cohesion is indeed algebraic connectivity, as explained earlier, but only for undirected and unweighted networks. 

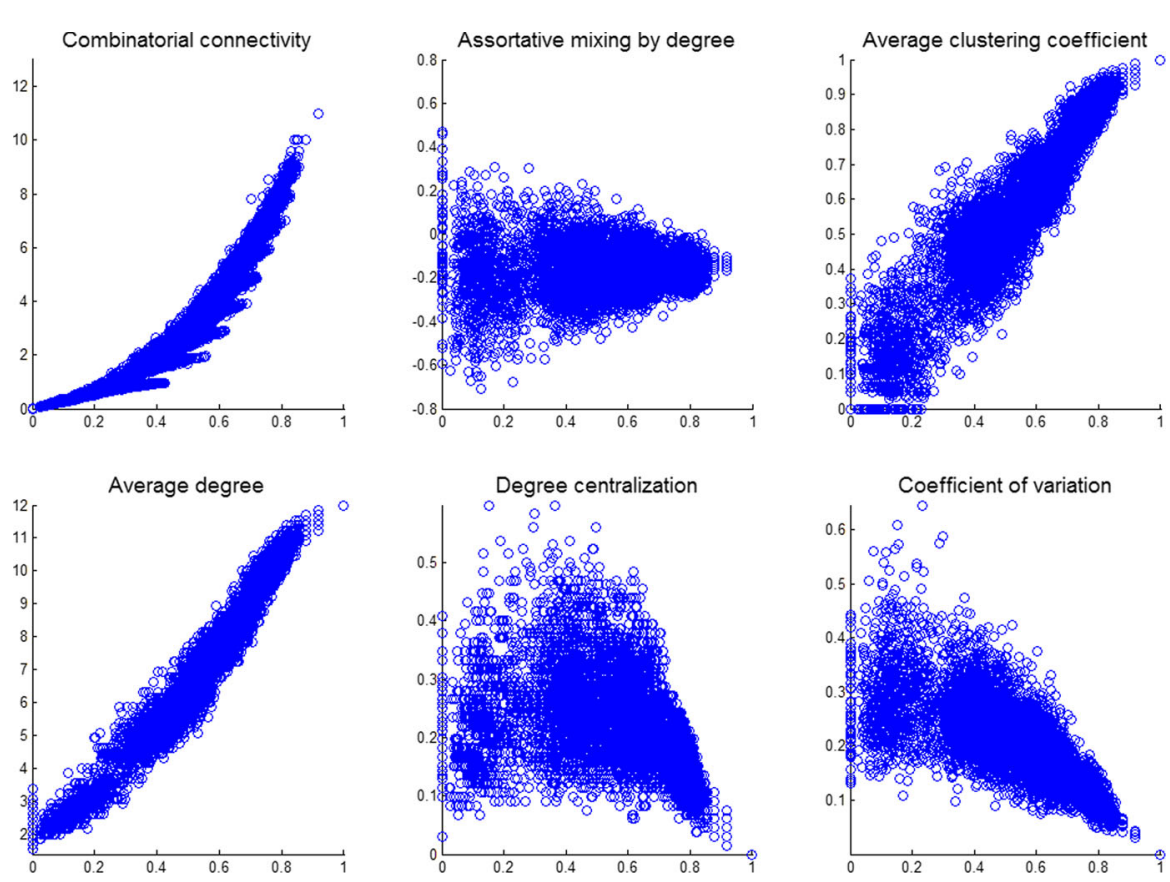

Fig. 2 Comparison of network cohesion (horizontal axis) and various related network statistics (vertical axis). Each panel is a scatter plot of cohesion $\kappa$ and corresponding measure for 10,000 randomly generated networks of $n=13$ nodes

\section{Closing comments}

In this paper we introduced the notion of network cohesion, a summary statistic that arises naturally in dynamic models of endogenous growth with (local) network externalities. In such frameworks, positive network cohesion is crucial for ensuring convergence to a balanced growth path and also determines the speed of convergence to this path. We argue that this statistic is in general useful and relevant for the dynamics of systems of variables that are defined in relative terms, i.e., as deviations from the average value of the variable. This is because network cohesion is defined as one minus the spectral radius of a matrix with elements that represent deviations of the importance of links from their averages. We also note that other summary statistics of networks that are mathematically related to cohesion (such as algebraic connectivity or spectral homophily) cannot be directly used for the characterization of dynamic systems with perpetual endogenous growth.

Open Access This article is distributed under the terms of the Creative Commons Attribution 4.0 International License (http://creativecommons.org/licenses/by/4.0/), which permits unrestricted use, distribution, and reproduction in any medium, provided you give appropriate credit to the original author(s) and the source, provide a link to the Creative Commons license, and indicate if changes were made. 


\section{References}

Acemoglu, D., Carvalho, V.M., Ozdaglar, A., Tahbaz-Salehi, A.: The network origins of aggregate fluctuation. Econometrica 80(5), 1977-2016 (2012)

Borgatti, S.P., Everett, M.G., Johnson, J.C.: Analyzing Social Networks. Sage Publications, Thousand Oaks (2013)

Cavalcanti, T. V. V., Giannitsarou, C.: Growth and human capital: a network approach. Econ. J (2015). doi:10.1111/ecoj.12337

DeGroot, M.H.: Reaching a consensus. J. Am. Stat. Assoc. 69, 118-121 (1974)

DeMarzo, P.M., Vayanos, D., Zwiebel, J.: Persuasion bias. Social influence, and unidimensional opinions. Q. J. Econ. 118(3), 909-968 (2003)

Diaconis, P., Saloff-Coste, L.: Geometric bounds for Eigenvalues of Markov chains. Ann. Appl. Probab. 1(1), 36-61 (1993)

Diaconis, P., Stroock, D.: Comparison techniques for random walk on finite groups. Ann. Prob. 21(4), 2131-2156 (1991)

Echenique, F., Fryer Jr., R.G.: A measure of segregation based on social interactions. Q. J. Econ. 122(2), 441-485 (2007)

Ertur, C., Koch, W.: Growth, technological interdependence and spatial externalities: theory and evidence. J. Appl. Econom. 22(6), 1033-1062 (2007)

Fiedler, M.: Algebraic connectivity of graphs. Czechoslov. Math. J. 23(98), 298-305 (1973)

Garcia-Peñalosa, C., Turnovsky, S.: Consumption externalities: a representative consumer model when agents are heterogeneous. Econ. Theory 37(3), 439-467 (2008)

Golub, B., Jackson, M.O.: How homophily affects the speed of learning and best-response dynamics. Q. J. Econ. 127(3), 1287-1338 (2012a)

Golub, B., and Jackson, M. O.: Does homophily predict consensus times? Testing a model of network structure via a dynamic process. Rev. Netw. Econ., 11(3), 9 (2012b). doi:10.1515/1446-9022.1367

Goyal, S.: Connections: An Introduction to the Economics of Networks. Princeton University Press, Princeton (2007)

Holroyd, M.: Synchronizability and connectivity of discrete complex systems. In: International Conference of Complex Systems, pp. 1-8 (2006)

Horn, R.A., Johnson, C.R.: Matrix Analysis. Cambridge University Press, Cambridge (1985)

Howe, E.C., Johnson, C.R.: Aggregation of Markov processes: axiomatization. J. Theor. Prob. 2(2), 201-208 (1989)

Jackson, M.O.: Social and Economic Networks. Princeton University Press, Princeton (2008)

Jiménez-Martínez, A.: A model of belief influence in large social networks. Econ. Theory 59(1), 21-59 (2015)

Konno, T.: Knowledge Spillover on Complex Networks, RIETI discussion paper series 10-E-002 (2010)

Landau, H.J., Odlyzko, A.M.: Bounds for Eigenvalues of certain stochastic matrices. Linear Algebra Appl. 38, 5-15 (1981)

Li, Q., Rus, D.: Global clock synchronization in sensor networks. IEEE Trans. Comput. 55(2), 214-226 (2006)

Melguizo, I.: Endogenous homophily and the persistence of disagreement. Mimeograph (2016, unpublished)

Mierau, J.O., Turnovsky, S.: Demography, growth, and inequality. Econ. Theory 55(1), 29-68 (2014)

Mitra, D., Weiss, A.: Analysis of delay-differential equations arising in communication network synchronization. In: Proceedings of the IEEE International Symposium on Circuits and Systems, pp 839-843 (1980)

Mohar, B.: The Laplacian spectrum of graphs. In: Alavi, Y., Chartrand, G., Oellermann, O.R., Schwenk, A.J. (eds.) Graph Theory, Combinatorics, and Applications, vol. 2, pp. 871-898. Wiley, Hoboken (1991)

Newman, M.E.J.: Networks: An Introduction. Oxford University Press, Oxford (2010)

Ray, D.: On the dynamics of inequality. Econ. Theory 29(2), 291-306 (2006)

Römer, K.: Time synchronization in ad-hoc networks. In ACM Symposium on Mobile Ad Hoc Networking and Computing MobiHoc 01 (2001) 